\title{
Antwort auf die Bemerkungen von Prof. Zuntz.
}

\author{
Von
}

Dr. B. Werigo aus St. Petersburg.

Soeben hat Prof. Zuntz einige kritische Bemerkungen auf meine Abhandlung: „Z $\mathrm{Z}$ r Frage $\mathfrak{i} b$ er die Wirkung des Sa uerstoffs a u f ie Koblensäureausscheidung in $d$ e $n L u n g e n^{\prime \prime}$ ) in diesem Archiv veröffentlicht. Er wendet mir nämlich ein, dass der Gasraum ${ }^{2}$ ), mit dem ich die Lungen bei meinen Versuchen in Verbindung liess, eine in den beiden Lungen verschiedenen Grad erreichende Diffusion der Kohlensäure nach diesem Raume hin gestattete. In der Wasserstofflunge musste die Kohlensäure schneller diffundiren, weil erstens die Diffusion im Wasserstoff schneller vor sich geht als im Sauerstoff, und zweitens, weil die aus der Sauerstofflunge diffundirte Kohlensäure Dank der beständigen Zufuhr des Sauerstoffs in die Lunge theilweise zuriickkehren musste. Der Verlust an Kohlensäure muss also in der Wasserstoff lunge bedeutend grösser sein als in der Sauerstofflunge. Dadurch sollen nach der Meinung von Prof. $\mathrm{Zuntz}$ die von mir gefundenen Differenzen der Kohlensäurespannung in den beiden Lungen mehr oder weniger erklärt werden.

Ich gebe gern zu, dass alle Vorgänge, wie sie Prof. $\mathrm{Zuntz}$ schildert, in der That stattfinden müssen. Ich war dessen vollkommen bewusst, als ich die Methode ersann, welche geeignet wäre, die in meirfer Abhandlung aufgeworfene Frage zu lösen. Aber es schien mir dann, dass die erwähnten Vorgänge der Diffu-

1) Dies Archiv Bd. 51. S. 321.

2) Diesen Gasraum, der sich von der Kanüle bis zu dem Wasserspiegel des Ventils $f$ (s. Fig. 2. S. 329) erstreokte, habe ich nicht gemessen, aber nach ungefährer Schätzung konnte er keineswegs mehr als die Hälfte des Lungeninhaltes betragen. 
sion nur einen Beweis a fortiori für die Richtigkeit meiner Schlüsse abgeben können. Ich habe auch jetzt diese Meinung nicht verändert. Es scheint mir nämlich, dass die Unterschiede der in den beiden Lungen stattfindenden Diffusion nur in dem Sinne wirken können, dass sie den KohlensäuregehaIt der aus der Sauerstofflunge herausgesaugten Gase nicht vergrössern, wie es Prof. $Z n n t z$ meint, sondern im Gegentheil verminder n.

Um meinen Gedaukengang schärfer auszudrücken, will ich den ganzen Athemraum meiner Versuche in drei Theile zerlegen, und nämlich: den Alveolen-, den Bronchial- und den üusseren Gasraum. Wenn wir jetzt die Vorgänge in jedem dieser Räume einzeln betrachten, so können wir sogleich ersehen, dass die Einwände von Prof. $Z$ untz in Bezug auf den Alveolenraum keine Anwendung finden. Die Bedingungen der Diffusion zwischen dem Blute und den Gasen dieses Raumes sind so ausserordentlich guinstig, dass die verhältnissmässig geringfïgige Diffusion zwischen diesem und dem Bronchialraume die Kohlensäurespannung nicht verändern kann. Wenn etwas Kohlensäure in den Bronchialraum entwichen ist, so muss dieselbe durch die Ausscheidung aus dem immer frisch zufliessenden Blute sofort ersetzt werden. Eine solche Diffusion könnte nur dann irgend welche störende Wirkung haben, wenn die Diffusionsbedingungen zwischen dem Alveolen- und Bronchialraume günstiger wären, als die zwischen den Blute und dem Alveolenranme. Es gilt hier aber bekanntlich gerade das entgegengesetzte Verhalten. Der zweite Umstand, den Prof. Zuntz hervorhebt, dass nämlich das zum Ersatz des verbrauchten Sauerstoffs nach der Sauerstofflunge hin strömende Gas etwas Kohlens:iure enthält, ist auch nicht im Stande, die Kohlensäurespannung. in dem Alveolenraume zu erböhen, weil dieses Gas jedenfalls weniger Koblensäure enthält als das in Alveolenraume befindliche. Wir könnten in der That die Sauerstofflunge anstatt mit reinem Sauerstoff mit einem Gemische von Sauerstoff und Kohlensäure anfüllen, wie wir es beim Gebrauch von Aërotonometern thun, ohne dabei die Resultate zu verändern. Es muss hier nur der Kohlensäuregehalt des die Lunge anfüllenden Gases nicht höher sein, als es der Kohlensäurespanuung des Blutes entspricht.

Aus den angeführten Betrachtungen können wir also zu dem Schlusse gelangen, dass die Kohlensäurespannung im Alveolenrame beider Lungen bei meiner Versuchsanord- 
nung genau der Kohlensäurespannung des circulirenden Blutẹs entsprechen musste ${ }^{1}$ ).

Wenn wir jetzt zu der Betrachtung der in den Bronchialräumen beider Lungen vor sich gehenden Vorgänge uibergehen, so ersehen wir leicht, dass die von Prof. $\mathrm{Zuntz}$ hervorgehobenen Unterschiede in den Diffusionsbedingungen einen Unterschied in dem Kohlensäuregehalte bedingen müssen: in der Wasserst of flunge, wo die Bedingungen der Diffusion zwischen dem Alveolen- und Bronchialraume viel günstiger sind, muss die Kohlensäure sich in einer grösseren Menge ansammeln als in der Sanerstofflunge. Es kann sogar, besonders bei den eine lange Zeit fortgesetzten Versuchen, der Fall vorkommen, wo die Kohlensäurespannung in dem Bronchialraume der Wasserstofflunge denselben Werth haben wird, wie in dem Alveolenraume, während in der Sauerstofflunge wegen der beständigen Strömung des Sauerstoffs nach der Lunge bin ein solcher Fall unmöglich erscheint.

In dem äusseren Gasraume miissen selbstverständlich dieselben Verhältnisse stattfinden, wie in dem Bronchialraume. Da wir aber beim Herausnehmen der Gasproben diesen Raum von den übrigen durch die Anlegung der Klemmen $s$ (s. Fig. 2 meiner Ab-

1) Bei den angeführten Auseinandersetzungen ging ich von dem Gesichtspunkte aus, dass die Ausgleichung der Spannungsdifferenzen zwischen dem Blute und den im Alveolenraume befindlichen Gasen fast momentan vor sich geht, d. h. von der jetzt allgemein anerkannten Ansicht, der auch Prof. $\mathrm{Zuntz}$ steuert. $\mathrm{Da}$ ich in meiner Arbeit einige Gründe anführe, welche mich glauben lassen, dass eine solche Schnelligkeit etwas übertrieben ist, so unuss ich zugeben, dass von diesem Standpunkte sowohl die Diffusion zwischen dem Alveolen- und Bronchialraume, als auch das theilweise Zurückkehren der schon im Bronchialraum abgeschiedenen Kohlensäure eine wenn auch geringe Wirkung haben kann. Diese Wirkung Inuss sich nämlich darin äussern, dass das Gleichgewicht zwischen der Kohlensäurespannung im Blute und im Alveolenraume sich in der Sauerstofflunge etwas früher einstellen muss, als in der Wasserstofflunge. Wenn wir also die Gasproben aus den Lungen herausnehmen zu der Zeit, wo dieses Gleichgewicht noch nicht vollkommen eingetreten ist, so muss der Kohlensäuregehalt in der Sauerstofflunge etwas höher ausfallen als in der Wasserstofflunge. Da dieser Einwand von Prof. Zuntz nicht gemeint sein konnte, so habe ich dessen im Texte keine Erwähnung gethan, um so mehr, als er nur für die Versuche von kurzer Dauer gelten und folglich keine principielle Bedeutung haben kann. 
handlung, S. 329) vollkommen trennen, so kann die Zusammensetzung der hier enthaltenen Gase keinen Werth für uns haben.

Wir haben also gesehen, dass die Kohlensäurespannung in den Alveolenräumen beider Lungen der des Blutes gleichkommen muss und dass der Kohlensäuregehalt des Bronchialraumes in der Sauerstofflunge unter allen gleichen Bedingungen niedriger ist, als der entsprechende Gehalt in der Wasserstofflunge. Da wir aber beim Herausnehmen der Gasproben zur Analyse den ganzen Inhalt des Bronchialraumes und einen Theil der im Alveolenraume befindlichen Gase heraussaugen, so müssen die für die Kohlensäurespannung in der Sauerstofflunge erhaltenen Zahlen etwas zu klein erscheinen. Wenn ich in der Wirklichkeit ein ganz entgegengesetztes Verhalten finde, so kann ich darin selbstverständlich nur einen Beweis a fortiori für das Vorhandensein der kohlensäureaustreibenden Wirkung des Sauerstoffs erblicken.

Ausser den oben erörterten principiellen Linwänden gregen meine Versuche wendet mir noch Prof. Zuntz ein, dass ich in einer flüchtigen Bemerkung (S. 342 meiner Abhandlung) die allgemein anerkannten Ansichten in Bezug auf die ungeheure Ausgleichungsschnelligkeit der Spannungsdifferenzen zwischen dem Blute und den in den Lungen befindlichen Gasen für etwas ibbertrieben erklärte. Ich habe mir diese Benerkung erlaubt, indem ich mich auf die Thatsache stiitzte, dass die Sauerstof'spannung in der Wasserstofflunge bei den eine kurze Zeit (30-60 Sec.) fortgesetzten Experimenten im Mittel nur 1,8\% betrug. Dagegen wendet mir Prof. Zuntz ein, dass bei den Bedingungen meiner Versuche das Blut weniger Sauerstoff enthalten musste als in der Norm, und folglich einen niedrigeren Werth der Sauerstoffspannumg anfweisen konnte. Indem ich in die Einzelheiten der Gründe, die Prof. Zuntz für die grössere Venösität des Blutes bei meinen Versuchen anführt, nicht näher eingehe, will ich nur auf deu Umstand aufmerksam machen, dass die unter allen gleichen Bedingungen erhaltenen Zahlen meiner dritten Versuchsreihe, wo die Versuche länger fortgesetzt wurden, eine nahezu normale Sauerstoffspannung in der Wasserstofflunge gezeigt haben, wie es der Leser aus den an den S. 350 und 352 angefïhrten Tabellen leicht entnehmen kann. Deshalb scheint es mir, dass meine flüchtige Bemerknng vollkommen berechtigt war. 